\title{
CHRISTOPH SCHLIER
}

Angular momentum dependence of unimolecular decay in a triatomic system 


\title{
Angular momentum dependence of unimolecular decay in a triatomic system
}

\author{
by CH. G. SCHLIER \\ Fakultät für Physik, Universität Freiburg, \\ D-7800 Freiburg, Germany
}

(Received 9 October 1986; accepted 29 July 1987)

\begin{abstract}
Classical trajectory calculations were performed to determine the lifetime $\tau$ of long-lived collision complexes in $\mathrm{H}^{+}-\mathrm{D}_{2}$ or $\mathrm{D}^{+}-\mathrm{HD}$ collisions. For the same complex characterized by total energy and total angular momentum, the lifetimes are independent of $(a)$ the kind of energy supply (i.e. translational or vibrational), and (b) the distribution of the three masses into projectile and target. It was found that $\tau(J)$ increases monotonously with $J$, first linearly, then faster, finally proportional to $\left(J_{\max }-J\right)^{-1}$. The shape of $\tau(J)$ can be understood in terms of statistical theory if the overall rotation is included in the assumed quasi-equilibrium.
\end{abstract}

\section{Introduction}

There are two conserved quantities in the dynamics of unimolecular decay, total energy, $E$, and total angular momentum, $J$. The decay rate constant $k$, and its reciprocal, the lifetime $\tau$, depend on both quantities, though the specific rate constant $k(E, J)$ and its $J$-dependence is seldom discussed. The probable reason is that measurements of this $J$-dependence do not exist, and only recently seem to become possible with laser techniques.

Moreover, most of today's discussion on $k(E, J)$ (e.g. in the textbooks, [1]) is limited to the influence of the barrier height changed by the addition of the centrifugal potential. For typical parameters, such changes are small, therefore large dependencies of $k$ on $J$ are not expected.

It was Quack and Troe, who have repeatedly mentioned $[2,3]$ that it could be interesting to know $k(J)$, and that there might be larger effects than anticipated. In the meantime a few calculations appeared in the literature. They have been done using statistical theories (statistical adiabatic channel model or RRKM theory) $[2,4,5]$, or classical trajectory computations [6-8]. The former have the advantage that they can include quantum effects if done elaborately, the latter that no approximation (beyond that of using classical mechanics) is involved, and that one can probe the assumptions of statistical theory, e.g. the exponential decay law. The price is high, though, since the computation times will be large due to 'chaotic' trajectories, which are an intrinsic property of long-lived complexes.

We compute our lifetimes from scattering trajectories. The range of $\tau$ accessible to computation is restricted on both sides: If $\tau$ is too long, the number of decays occurring during the time interval, in which the accuracy of the trajectories can be assured, becomes too small; this leads to bad statistics. If $\tau$ is too short, one runs into the problem of induction time. This is the time needed to form the complex into an entity which has 'forgotten' from which initial state it came. Induction times can 
be found from the trajectories by monitoring, e.g., the branching ratio of the decay into different channels. Only after this and similar properties are constant can one begin to determine the lifetime. Induction times are further discussed in $[8,9]$. Alternatively one could, of course, start the calculation in the interior of the complex with initial conditions taken according to a microcanonical ensemble. However, this leads to other problems: Apart from the difficulty to implement the correct initial conditions, and the somewhat arbitrary boundary of the complex (the 'critical configuration'), it implies the assumption that the whole phase space within that boundary belongs uniformly to long-lived complexes. This again is generally not true, since 'bound states in the continuum' and states which dissociate directly must be excluded.

\section{Method}

We have calculated classical trajectories which simulate scattering events at fixed total angular momentum $J$ (in units of $\hbar$ ), where either $J=L$ (orbital angular momentum) and $j=0$ (target rotational angular momentum), or $J=j$ and $L=0$. The system was $\mathrm{HD}_{2}^{+}$, and we used the DIM-potential for $\mathrm{H}_{3}^{+}$[10], which is good enough for the purpose. Both initial channels, $\mathrm{H}^{+}+\mathrm{D}_{2}$ as well as $\mathrm{D}^{+}+\mathrm{HD}$ were probed. The total energy was $0.5 \mathrm{eV}\left(\approx 1 / 10\right.$ of the well depth), and for the $\mathrm{H}^{+}+\mathrm{D}_{2}$ channel it was supplied either as translational energy only, or as $0.1 \mathrm{eV}$ translational plus $0.4 \mathrm{eV}$ vibrational energy (vibrational quantum number $v=0.54$ ). The trajectories were integrated with a variable stepsize Gear integrator for $800 \mathrm{fs}$. The use of double precision was imperative. An average trajectory needed 10 min on a PE 3220 computer (similar to a VAX 750).

There are 3 methods to determine the lifetime from the trajectory data. Two of them have been discussed before [8], and will only be mentioned here. In the first we fit a histogram of the number of living complexes $v$ s. time to an exponential. Early times must be excluded because not even all complexes have been formed. In the second method we fit a histogram of the time intervals between the first and last 'minimum exchange' of each trajectory, i.e. the first and last time, where $R_{\min }=$ $\operatorname{Min}\left(R_{A B}, R_{B C}, R_{C A}\right)$ changes its identity. This is similar to using the first and last interior turning points of the reaction coordinate. Both methods agree in their results to within statistical errors.

For the two cases with very long lifetimes another method had to be used: One divides the observation time into time intervals $\Delta t$ which are small enough that the number of decays per interval of a set of $N_{0}$ complex trajectories is either one $\left(n_{1}\right.$ intervals) or zero ( $n_{0}$ intervals). Then the maximum likelihood method estimate of $\tau$ and of its error is

$$
\tau=N_{0} \frac{n_{0}}{n_{1}} \Delta t\left(1 \pm 1 / \sqrt{ } n_{1}\right) .
$$

In addition to the lifetimes we show in figure 2 the probability $P_{c}$ of complex formation, and in figure 3 the average final fractional energy disposal expressed by $f_{\text {trans }}=E_{\text {trans }}^{\prime} / E, f_{\text {vib }}=E_{\text {vib }}^{\prime} / E$, and $f_{\text {rot }}=E_{\text {rot }}^{\prime} / E$, where the primed entities are those after the collision. These results are based only on trajectories, which have performed more than 7 minimum exchanges and can, therefore, be considered to be long-lived statistical complexes (cf. [11]). 


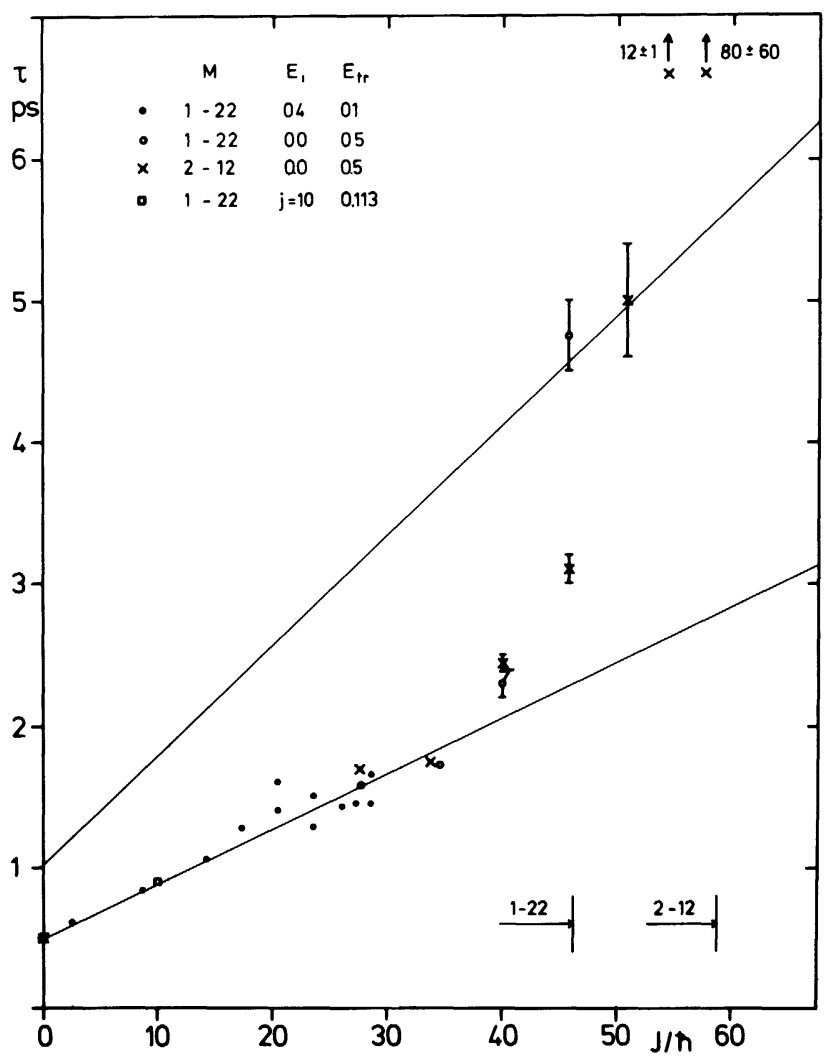

Figure 1. Lifetime versus total angular momentum of complexes formed with different initial conditions at a common total energy of $0.5 \mathrm{eV}$. , masses $1-22, E_{\text {trans }}=0.1 \mathrm{eV}$, $E_{\text {vib }}=0.4 \mathrm{eV} ; O$, masses $1-22, E_{\text {trans }}=0.5 \mathrm{eV}, E_{\text {vib }}=0 ; \times$, masses $2-12, E_{\text {trans }}=$ $0.5 \mathrm{eV}, E_{\text {vib }}=0 ; \square$, masses $1-22, E_{\text {trans }}=0.113 \mathrm{eV}, E_{\text {vib }}=0, E_{\text {rot }}=0.387 \mathrm{eV}(j=10)$. Note the two lifetime values which are off scale. Values of $J_{\max }$ are indicated at the bottom for the decay into two mass combinations. Typical statistical errors for low $J$ are \pm 0.03 ps increasing to larger values as indicated.

\section{Results}

Our main results are contained in figures $1-3$. From these we can read the following.

(a) Lifetimes (in contrast to complex formation probabilities and fractional energy disposals) are very dependent on total angular momentum. Let us call the maximum angular momentum with which the complex may classically decay at the given energy $J_{\max }$ (different for each channel). At $0.5 \mathrm{eV} J_{1_{\max }}$ for decay into $\mathrm{H}^{+}+\mathrm{D}_{2}$ is 47 , while $J_{2 \max }$ for decay into $\mathrm{D}^{+}+\mathrm{HD}$ is 59 . Then from $J=0$ to about $J=35 \approx 0.75 J_{1 \max }$ the lifetime increases linearly with $J$. From there on it can be nicely fitted by the formula

$$
\tau(J)=\frac{43.9}{J_{2 \max }-J} \text { ps. }
$$

This means that $k(J)$ decreases linearly with $J \rightarrow J_{2 \max }$. A similar formula fits, e.g., also to calculations by Troe [4] who using a statistical model computes the decay of 


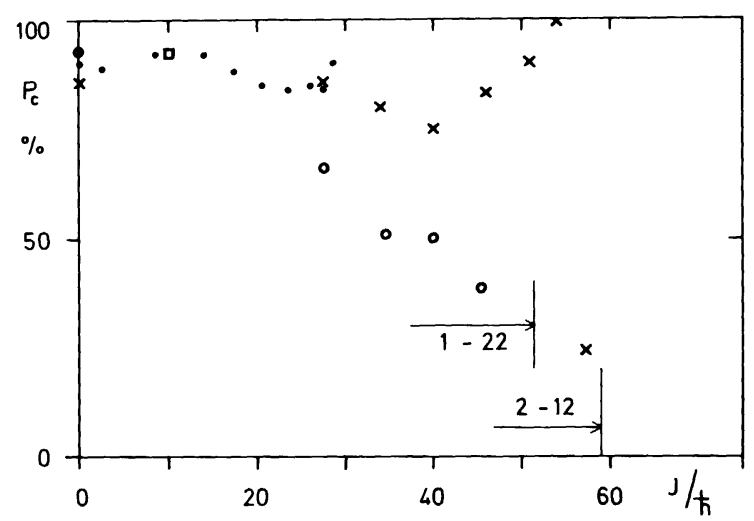

Figure 2. Complex formation probability versus total angular momentum. See figure 1 for codes marking initial conditions. Statistical errors of $P_{\mathrm{c}}$ are $\left(\boldsymbol{P}_{\mathrm{c}} \cdot\left(1-\boldsymbol{P}_{\mathrm{c}}\right) / 1000\right)^{1 / 2}$, i.e. $1-3$ per cent.

$\mathrm{H}_{2} \mathrm{CO}$ into $\mathrm{H}_{2}+\mathrm{CO}$. On the contrary, Troe's $\tau(J)$ for $\mathrm{H}_{2} \mathrm{CO} \rightarrow \mathrm{H}+\mathrm{HCO}$ is not even monotonous, and only for the highest $J$ seems to fit to equation 2 .

(b) The lifetime, though it depends heavily on $E$ and $J$, does not depend on whether the complexes are formed in collisions of $\mathrm{H}^{+}+\mathrm{D}_{2}$ or $\mathrm{D}^{+}+\mathrm{HD}$. Note, however, that the average lifetime

$$
\tau=\frac{\sum(2 J+1) P_{c}(J) \tau(J)}{\sum(2 J+1) P_{c}(J)},
$$

where $P_{\mathrm{c}}$ is the complex formation probability, will depend on the channel and on the kind of energy supply $\left(E_{\text {trans }}\right.$ or $\left.E_{\text {vib }}\right)$, since the distributions $P_{\mathrm{c}}(J)$ depend on both. The average lifetimes determined with the procedures of [8] are: $1.10 \pm 0.02 \mathrm{ps}$ for masses $1-22, E_{\text {trans }}=0.1 \mathrm{eV}, E_{\text {vib }}=0.4 \mathrm{eV} ; 1.45 \pm 0.05 \mathrm{ps}$ for $1-22, E_{\text {trans }}=0.5 \mathrm{eV}, E_{\mathrm{vib}}=0$; and $2.7 \pm 0.3 \mathrm{ps}$ for $2-12, E_{\text {trans }}=0.5 \mathrm{eV}, \mathrm{E}_{\mathrm{vib}}=0$.

(c) The lifetime is also independent of the source of the energy supplied to the complex, i.e. whether it came mainly from translation or vibration. This is also true

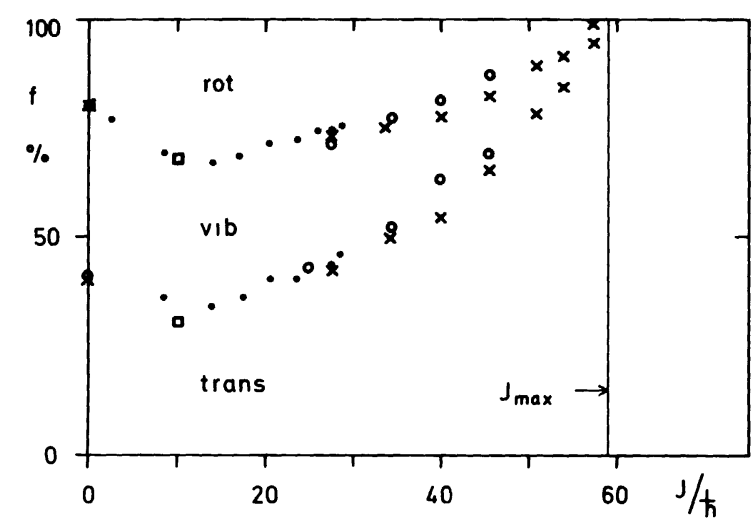

Figure 3. Fractional energy disposal from unimolecular decay versus total angular momentum. Initial conditions are marked as in figure 1 . Note that the $f s$ are averages of broad distributions: The typical relative rms halfwidth, e.g., of the rotational distributions is $\Delta f_{\text {rot }} / f_{\text {rot }} \approx 0.8$. 
(see the point at $J=10$ ) if most energy ( 77 per cent) and all angular momentum is supplied by rotation of the target.

(d) Complex formation probabilities at this energy are uniformly high ( $\approx 90$ per cent) for $J \lesssim J_{i \max } / 2(i=1,2)$, then drop to zero at the maximum $J$ allowed for each initial condition. (This is $J_{i \max }$ only if $E_{\text {trans }}=E, E_{\mathrm{vib}}=E_{\text {rot }}=0$.) Expressed by $P_{\mathrm{c}}(J)$ the complex formation cross-section is

$$
\sigma_{\mathrm{c}}=\pi k^{-2} \sum(2 J+1) P_{\mathrm{c}}(J)
$$

It can easier be determined by Monte Carlo integration over the impact parameter $b$. The values obtained for the three cases mentioned under item $(b)$ are 6.18 $\pm 1.0 \AA^{2}, 22 \cdot 7 \pm 0.7 \AA$, and $30.9 \pm 0.7 \AA^{2}$. These values amount to 89,65 , and 81 per cent of $\pi b_{\max }^{2}$, where $b_{\max }$ is the largest value of $b$ for which the classical centrifugal barrier can be overcome and a complex formed.

(e) The fractional energy disposal of the decay, i.e. $f_{\text {rot }}=E_{\text {rot }}^{\prime} / E$, etc., assumes its 'statistical' value of $f_{\text {rot }}=f_{\text {vib }}=f_{\text {trans }}=1 / 3$ only between $J \approx 10$ and $J \approx 20$. For $J=0 f_{\text {rot }} \approx 20$ per cent, but $f_{\text {vib }} \approx f_{\text {trans }}$ as expected. For $J \rightarrow J_{\max }$ the requirement that all available energy be used to exit over the centrifugal wall leads to $f_{\text {vib }} \rightarrow 0$, $f_{\text {rot }} \rightarrow 0$ in each channel. This is obvious from figure 3 for total angular momenta $>47$, where only the channel $2-12$ remains open for decay. For $J \lesssim 47$ the fractions $f$ are mainly determined by the open channel $2-12$, and not so much by the closing channel 1-22.

$(f)$ We restrain from plotting $j^{\prime}$, the average angular momentum of the product diatom, but note that it is between 3 and 5 for $J$ below 45. For $J \rightarrow J_{i \max }$ it must, of course, vanish in each channel. For almost all conditions therefore $j^{\prime} \ll J$. This is the consequence of the large moment of inertia of the transition state in comparison with that of the product diatom.

\section{Discussion}

Further understanding of the behaviour of $\tau(J)$ can be attempted on two levels, that of dynamics, and that of statistics. In both cases it will be useful to distinguish the case of small $J$ (i.e. $B^{\ddagger} J^{2} \ll E, B^{\ddagger}$ being an averaged rotational constant of the transition state), and that of large $J$ (i.e. $J \rightarrow J_{\max }$, where $J_{\max }$ is the limiting total angular momentum of the last closing decay channel, here with masses 2-12).

On the dynamical level we have been unable to find any argument predicting a linearly increasing $\tau(J)$ (or any other specific behaviour) for small $J$. For $J \rightarrow J_{\max }$, on the other hand, the dynamics seems to be quite clear: At given $E$ and $J$ the minimum amount of energy fixed in overall rotation is given by the minimal effective potential [12]

$$
U_{J}^{\min }(R)=\min _{r, \gamma}\left[U_{J}(R, r, \gamma)\right]
$$

where

$$
U_{J}(R, r, \gamma)=V(R, r, \gamma)+J^{2} / 2 I
$$

and

$$
I=m_{A B C} R^{2}+m_{B C} r^{2}
$$

Here we have used Jacobi coordinates for the atom-diatom system, $r$ being the diatom distance, $R$ the distance from the atom to the centre of mass of the diatom, 
and $\gamma$ the angle between the vectors $\mathbf{R}$ and $\mathbf{r} \cdot m_{B C}$ is the reduced mass of the diatom, and $m_{A B C}$ that of the atom-diatom system. $U_{J}^{\min }$ replaces the usual effective potential of atom-atom collisions in the triatomic case. The classical threshold energy for unimolecular decay at given $J, E(J)$, or the largest angular momentum allowing decay at given energy, $J_{\max }(E)$, are given exactly by equating equation (5) to $E$. From equation $(6 b)$ one sees that the $I$ to be used is the rigid moment of inertia (even for a very 'loose' complex), and the minimization of $U_{J}$ means that (classically) $r$ must be relaxed to its equilibrium value at each given $R$. It follows that, when a decay channel closes as a function of $J$ (at given $E$ ), the conformation representing the 'bottleneck' is a very singular one, whose phase space contracts to zero in all coordinates except the reaction coordinate. So it seems to be obvious that for $J \rightarrow J_{\max }$ the specific rate $k(E, J)$ must drop to zero at least linearly, which is in accord with our calculations.

As a consequence from this dynamics, any internal energy of the product diatom near threshold must come from post-barrier interaction. Figure 3 shows that for $J \rightarrow J_{\max }$ this interaction is very small indeed. For the last point shown $\left(J_{\max }-J=\right.$ $1.4)$ the average internal energy of the two completed trajectories is only 6 per cent of $E$.

Next we want to discuss this same behaviour in terms of statistical theory $[1-3,13]$. We start from the general formula

$$
k(E, J)=\frac{W^{\ddagger}(E J)}{h \rho(E J)},
$$

where $W^{\ddagger}$ is the number of states below $E$ at the transition state, and $\rho$ is the density of states of the excited molecule at the energy $E$.

Note that we apply traditional quantum language to equation (7), since it is much simpler to use, although the content of this equation in our context is strictly classical. This can, e.g., be seen from [13], and does not contradict the appearance of $h$ in the formula, whose reason is that we measure $k(E, J)$ not in units of phasespace-volume-in-the-reaction-coordinate per second, but rather in units of particles (i.e. wave packets having a volume $h$ ) per second.

Traditionally the overall rotation is treated as three 'adiabatic' degrees of freedom, i.e. one considers only energetic effects of introducing $J$ as a conserved quantity. The energy of the excited molecule is raised (cf. figure 4) by

$$
E_{\text {rot }}=B J^{2} \text {, }
$$

that of the transition state by

$$
E_{\text {rot }}^{\ddagger}=B^{\ddagger} J^{2} \text {. }
$$

Here the usual rigid spherical top approximation has been made, understanding that $B$ and $B^{\ddagger}$ must be considered as averages over the three coordinates and over vibrations. Using a common energy zero, and introducing the well depth $D$ of the nonrotating molecule this leads to

$$
k(E, J)=\frac{W^{\ddagger}\left(E_{\mathrm{vib}}^{\ddagger}\right)}{h \rho\left(E_{\mathrm{vib}}\right)},
$$

with

$$
E_{\mathrm{vib}}=E+D-E_{\mathrm{rot}},
$$




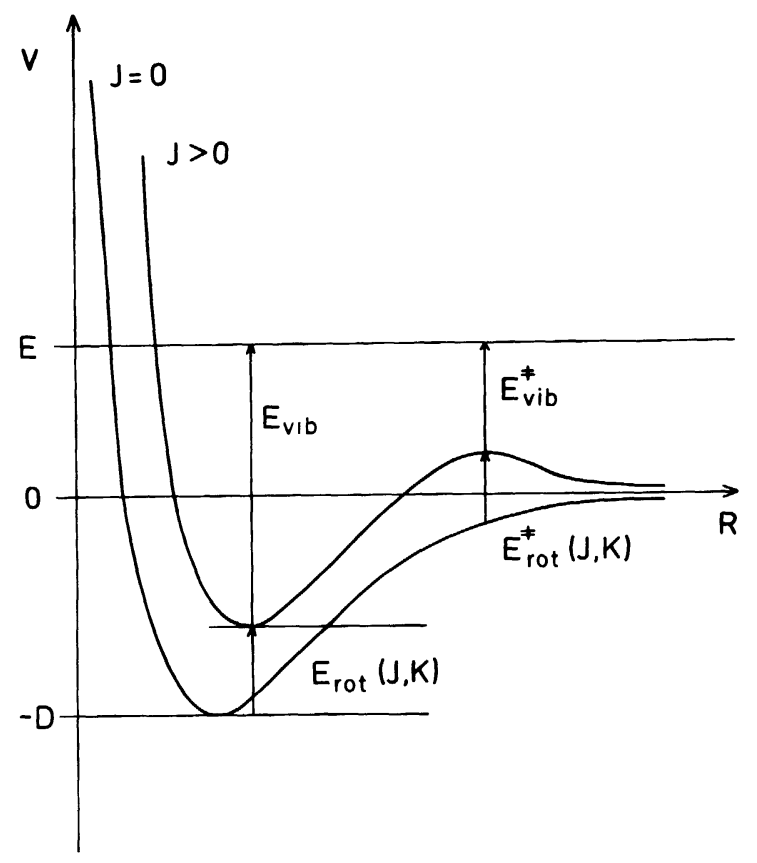

Figure 4. Schematic drawing of the effective potentials and energies involved in statistical theory.

and

$$
E_{\mathrm{vib}}^{\ddagger}=E-E_{\mathrm{rot}}^{\ddagger},
$$

where now $W$ and $\rho$ count only vibrational states at the energy of the argument. In a triatomic molecule both, numerator and denominator, are quadratic functions of their argument [14], which leads to

$$
\begin{aligned}
\tau(E, J) & =\frac{1}{k(E, J)}=\frac{\left(E+D-B J^{2}\right)^{2}}{\left(E-B^{\ddagger} J^{2}\right)^{2}}, \\
& \simeq \tau(E, 0)\left\{1+2 \frac{B^{\ddagger} J^{2}}{E}-2 \frac{B J^{2}}{E+D}+\ldots\right\},
\end{aligned}
$$

if $J$ is small. Therefore, starting from $J=0, \tau(E, J)$ increases with $J$ as long as

$$
E<D \frac{B^{\ddagger}}{B-B^{\ddagger}},
$$

which holds in our system for all energies at which complexes are formed. This quadratic dependence on $J$ is in contradiction to the trajectory calculations, even if the sign of the effect is correct. This situation is not changed if better approximations to the rotational energy are used, since it will always be quadratic in $J$. We conclude (in accord with a simpler statement made in an earlier paper [8]) that the statistical theory combined with the assumption of adiabatic overall rotation can not explain the trajectory data.

It was Quack and Troe who insisted $[2,3]$ that the hypothesis of adiabaticity of the $K$-rotor might be wrong, since Coriolis forces will couple it to the vibrational 
degrees of freedom. Assuming that this coupling is large enough we have now

$$
k(E, J)=\frac{\sum W^{\ddagger}\left(E_{\mathrm{vib}}^{\ddagger}\right)}{h \sum \rho\left(E_{\mathrm{vib}}\right)},
$$

where

$$
\begin{aligned}
& E_{\mathrm{vib}}=E+D-E_{\mathrm{rot}}(J, K) \simeq E+D-B J^{2}-(C-B) K^{2}, \\
& E_{\mathrm{vib}}^{\ddagger}=E-E_{\mathrm{rot}}^{\ddagger}(J, K) \simeq E-B^{\ddagger} J^{2}-\left(A^{\ddagger}-B^{\ddagger}\right) K^{2},
\end{aligned}
$$

and the sums are taken from $K=-J$ to $K=J$ with the further condition that the vibrational energies must be positive. Here we have approximated the excited molecule and the transition state as oblate resp. prolate symmetric tops. Whereas in the adibatic case $W^{\ddagger}(J)$ and $\rho(J)$ are monotonic decreasing functions of $J$, now both start from $J=0$ with a linear increase, which classically is proportional to $2 J$ (and quantum-mechanically would be $\sim 1+2 J$ ). However, this increase is soon cut off by the exhaustion of the available energy. Since $A^{\ddagger} \gg B>C$ this happens for $W^{\ddagger}$ at smaller $J$ than for $\rho$. In this manner both functions acquire a flat-peaked shape. They drop to zero at some limiting $J$, which obviously must be smaller for $W^{\ddagger}$ than for $\rho$. Such curves have been published at several occasions, see, e.g. [15] for $\mathrm{H}_{3}^{+}$ and [5].

There is no simple way to evaluate equation (12) non-numerically. The simplifying assumption that $\rho$ is proportional to $2 J$ for a much greater $J$-range than $W^{\ddagger}$ would lead to $\tau \sim 2 J$, which we do not observe from the trajectories. Apparently the long linear increase of $\tau(J)$ observable in figure 1 , roughly $\sim(1+0.077 J)$, comes from a subtle interaction between the functions $W^{\ddagger}(J)$ and $\rho(J)[16]$. One should, however, notice that this long linear increase is incompatible with statistical theory if the $K$-rotor behaves adiabatically, and that it, therefore, gives strong evidence that rovibronic coupling must be included in a correct statistical theory of unimolecular decay.

For large $J$ the behaviour of $\tau(E, J)$ will be dominated by that of $W^{\ddagger}(E, J)$, which goes to zero at $J=J_{\max }$. Again, there is no particular reason why the decrease of $k(E, J)$ should be linear as observed within the error (in this case large) of the calculation. So we must again conclude that this linearity is more or less accidental, while the insight from dynamics that $\tau \rightarrow \infty$ for $J \rightarrow J_{\max }$ is confirmed by statistical theory.

\section{Conclusions}

We have shown in a classical model calculation, that the lifetime of chemically activated triatomic complexes depends strongly on total angular momentum. Our model system is $\mathrm{H}_{3}^{+}$, but the results can be generalized by scaling of masses, energies and angular momenta to other systems with similar potential shapes. The dependence on potential shapes is being investigated [19], it seems to be quite modest. In the case treated, starting from $J=0$ there is a long linear increase of $\tau(J)$ with $J$, which then accelerates: eventually $\tau$ diverges to infinity for $J \rightarrow J_{\max }(E)$, the largest $J$ allowing decay. In this region $k(E, J)=$ const $/\left(J_{\max }-J\right)$ is a good approximation to the data. The initial linear increase of $\tau(J)$ with $J$ provides strong evidence for the influence of rovibronic coupling, i.e. the non-adiabaticity of the $K$-rotor, which has been emphasized by Quack and Troe since 1974 [2]. Further 
efforts to derive $\tau(J)$ from exact phase space calculations of $W^{\ddagger}$ and $\rho$ are under way, and we hope to publish them soon [16]. They show that statistics reproduces the dynamical lifetimes to better than 25 per cent in the $\mathrm{H}_{3}^{+}$system.

Helpful discussions witk M. Quack and D. Gerlich are gratefully acknowledged. This work was supported by the Deutsche Forschungsgemeinschaft.

\section{References}

[1] FoRst, W., 1973, Theory of Unimolecular Reactions (Academic Press), Chap. 7. Robinson, P. J., and Holbrook, K. A., 1972, Unimolecular Reactions (Wiley-Interscience), Chap. 4, 10.

[2] QuaCK, M., and Troe, J., 1974, Ber. Bunsenges. phys. Chem., 78, 240; 1975, Ibid., 79, 170.

[3] QuaCK, M., and Troe, J., 1981, Theor. Chem. (N.Y.) B, 6, 199, especially § D.3.b.

[4] Troe, J., 1984, J. phys. Chem., 88, 4375.

[5] Wardlaw, D. M., and Marcus, R. A., 1985, J. chem. Phys., 83, 3462.

[6] Miller, J. A., and Brown, N. J., 1982, J. phys. Chem., 86, 772.

[7] BABCOCK, L. M., and ThOMPSON, D. L., 1983, J. chem. Phys., 78, 2394.

[8] SChlier, CH., and Vix, U., 1985, Chem. Phys., 95, 401. Note that due to a mistake the lifetimes (in fs) of tables 2-4 must be multiplied by 10 .

[9] BRASS, O., and SCHLIER, CH. (in preparation).

[10] Preston, R. K., and Tully, J. C., 1971, J. chem. Phys., 64, 4297.

[11] SChlier, CH., 1983, Energy Storage and Distribution in Molecules, edited by J. Hinze (Plenum Press).

[12] Pollak, E., 1987, J. chem. Phys., 86, 1645.

[13] Nixitin, E. E., 1974, Theory of Elementary Atomic and Molecular Processes in Gases (Clarendon Press), especially pp. 11f. and 242f.

[14] For a very loose transition state the exponent could be as low as 1.5 but [8] shows that for our case it is near 2.0.

[15] QuACK, M., 1979, J.phys. Chem., 83, 150.

[16] This has meanwhile been verified by numerically exact computations of $W^{\ddagger}(E, J)$ and $\rho(E, J)[17]$. For the method (Monte Carlo integration) see also [18] and [5].

[17] Berblinger, M., and Schlier, Ch. (to be published).

[18] Berblinger, M., Pollak, E., and Schlier, Ch., J. chem. Phys. (to be published).

[19] BRASS, O., and SChlier, CH., J. chem. Phys. (in the press). 